\title{
PHYSICS OF VIBRATING AIRFOILS AT LOW REDUCED FREQUENCY
}

\author{
Almudena Vega \\ School of Aeronautics \\ Universidad Politécnica de Madrid \\ 28040 Madrid, Spain \\ e-mail: almudena.vega@upm.es
}

\author{
Roque Corral* \\ Technology and Methods Department \\ Industria de TurboPropulsores S.A. \\ 28830 Madrid, Spain \\ e-mail: roque.corral@itp.es
}

\begin{abstract}
The unsteady aerodynamics of low pressure turbine vibrating airfoils in flap mode is studied in detail using a frequency domain linearized Navier-Stokes solver. Both the travelling-wave and influence coefficient formulations of the problem are used to highlight key aspects of the physics and understand different trends such as the effect of reduced frequency and Mach number. The study is focused in the low-reduced frequency regime which is of paramount relevance for the design of aeronautical low-pressure turbines and compressors. It is concluded that the effect of the Mach number on the unsteady pressure phase can be neglected in first approximation and that the unsteadiness of the vibrating and adjacent airfoils is driven by vortex shedding mechanisms. Finally a simple model to estimate the work-percycle as a function of the reduced frequency and Mach Number is provided. The edge-wise and torsion modes are presented in less detail but it is shown that acoustic waves are essential to explain its behaviour. The non-dimensional work-per-cycle of the edge-wise mode shows a large dependence with the Mach number while in the torsion mode a large number of airfoils is needed to reconstruct the work-per-cycle departing from the influence coefficients.
\end{abstract}

\section{INTRODUCTION}

Aeroengine low pressure turbines (LPTs) are made of very slender and thin airfoils because their weight and cost have a large

\footnotetext{
*Also associate professor at the Department of Engine Propulsion and Fluid Dynamics of the School of Aeronautics, UPM
}

impact on the engine (about 20\% of the total weight and 15\% of the total cost). The natural frequencies of the blades are very low and, as a consequence, LPT assemblies are prone to flutter. Nowadays flutter may become a dominant constraint on the design of modern LPTs precluding the use of more efficient aerodynamic configurations $[1,2]$.

The unsteady aerodynamics of low-pressure turbine rotor blades and stator vanes associated to the airfoil vibration is nowadays routinely analyzed within the design loop of the aeroengine companies and it has been the subject of dedicated experiments $[3,4,5]$. The standard application of all these efforts is the derivation of the aerodynamic stability of the rotor blades and the quantification of the aerodynamic damping, which is the result of the application of the unsteady pressures on the airfoil displacements.

Little attention has been paid however to the understanding of vibrating airfoil aerodynamics since this is not a figure of merit in itself for the aeroelastic analyst. Designers obtain and use aerodynamic data from numerical tools but often they do not even inspect the unsteady flow field once the methods are productionised, since they are really just interested in the aerodynamic damping. As a direct consequence of using aerodynamic codes as black boxes little understanding has been gained in the last years about the physics of these type of flows and although there are several trends which are well known the physics which is behind is not really well understood. On the other hand vibrating cascade experiments are scarce, and performed well apart in time and in different institutions[6] with different techniques and therefore are not well suited for conceptual studies.
Copyright (C) 2013 by ASME 
This paper first reviews the mathematical dependence of the unsteady aerodynamics with the reduced frequency, then revisits the flow physics and work-per-cycle at low reduced frequency, then a typical low-pressure turbine airfoil is used to investigate the dependence of the work-per-cycle and the influence coefficients with the reduced frequency in the flap mode and in less detail for the edge-wise and torsion modes. Finally some conclusions are drawn.

\section{NOMENCLATURE}

\begin{tabular}{|c|c|}
\hline$c$ & Axial chord \\
\hline $\mathrm{h}$ & Span-wise length of the cascade \\
\hline IBPA & Inter-blade Phase Angle \\
\hline IC & Influence Coefficient \\
\hline$k$ & $=\frac{\omega c}{U_{\text {exit }}}$ Reduced frequency \\
\hline LPT & Low-Pressure-Turbine \\
\hline$M$ & Mach number \\
\hline PS & Pressure side \\
\hline$R e$ & Reynolds number \\
\hline$s$ & Spacing \\
\hline$t$ & Time \\
\hline $\mathrm{SS}$ & Suction side \\
\hline TW & Travelling-Wave \\
\hline$U_{\text {ref }}$ & Characteristic velocity of the mean flow \\
\hline$\delta$ & Vibration amplitude \\
\hline$\rho$ & Density \\
\hline$\phi$ & Phase of the work-per-cycle curve \\
\hline$\sigma$ & Inter-blade phase angle \\
\hline$\tau$ & Non-dimensional time \\
\hline$\omega$ & Angular frequency \\
\hline & Super-scripts \\
\hline$\hat{\imath}$ & Fourier transform \\
\hline & Sub-scripts \\
\hline c & Characteristic \\
\hline $\mathrm{e}$ & Exit \\
\hline 0 & Stagnation conditions \\
\hline$\Pi_{R}$ & $=\frac{p_{\text {exit }}}{P_{0}}$ Pressure ratio \\
\hline$v$ & flow velocity \\
\hline$\pi$ & pi number \\
\hline $\mathrm{h}$ & blade height (slide width for 2D cases) \\
\hline
\end{tabular}

\section{GOVERNING EQUATIONS}

The time-dependent Navier-Stokes equations of an ideal gas in conservative and compact form may be written as :

$$
\frac{\partial U}{\partial t}+\nabla \cdot F_{c}(U)=\nabla \cdot F_{v}(U)
$$

where $U=\{\rho, \rho v, \rho E\}^{T}$ is the vector of conservative variables and $F_{c}$ and $F_{v}$ the convective and viscous fluxes respectively. Without loss of generality the simplest boundary conditions for a two-dimensional airfoil cascade could be written as $P_{t}=P_{0}$, $T_{t}=T_{0}$ and $\alpha=\alpha_{i n}$, at the inlet, $p=p_{\text {exit }}$ at the exit and null velocity, $v=0$, and heat flux, $\partial T / \partial n=0$, at the airfoil's solid walls, periodic boundary conditions are also enforced at the lateral walls.

The above equations may be non-dimensionalised using $p_{c}=P_{0}$, $T_{c}=T_{0}$ and $v_{c}^{2}=2 c_{p} T_{0}\left(1-\left(p_{\text {exit }} / P_{0}\right)^{(\gamma-1) / \gamma}\right)$ as the characteristic pressure, temperature and velocity respectively. The characteristic velocity used here is the isentropic exit velocity that may be readily computed from the boundary conditions. The airfoil chord, $c$, is used as the characteristic length, $L_{c}$, then choosing the residence time, $t_{R}=c / v_{c}$, as the characteristic time $t_{c 1}=t_{R}$, Eq. 1 may be written in non-dimensional form as

$$
\frac{\partial \tilde{U}}{\partial \tau_{1}}+\tilde{\nabla} \cdot F_{c}(\tilde{U})=\frac{1}{R e} \tilde{\nabla} \cdot F_{v}(\tilde{U})
$$

where $\tau_{1}=t / t_{R}$ is the non-dimensional time, $\tilde{x}=x / c$ the nondimensional distance and $\tilde{U}=\{\tilde{\rho}, \tilde{\rho} w, \tilde{\rho} \tilde{E}\}^{T}$ the vector of nondimensional conservative variables with $\tilde{\rho}=\rho / \rho_{0}, w=v / v_{c}$ and $E=\frac{1}{2 \gamma} \tilde{T} /\left(1-\Pi_{R}^{-(\gamma-1) / \gamma}\right)+\frac{1}{2} w^{2}$. The non-dimensional boundary conditions become $\tilde{P}_{t}=1, \tilde{T}_{t}=1$ and $\alpha=\alpha_{i n}$, at the inlet, $\tilde{p}=\Pi_{R}^{-1}$ at the exit and $w=0$ and $\partial \tilde{T} / \partial \tilde{n}=0$ at the airfoil's solid walls. It is important to highlight that the solution depends solely on the Reynolds number, that directly appears in Eq 2 and the pressure ratio, $\Pi_{R}$ or the exit Mach number that appears in the exit boundary condition.

For vibrating airfoils the solid wall boundary condition becomes:

$$
\text { at } \tilde{x}=\tilde{x}_{0}+\Delta \tilde{\mathbf{x}} \sin \left(k \tau_{1}\right) \quad w=\Delta \tilde{\mathbf{x}} k \cos \left(k \tau_{1}\right)
$$

while the governing equations remain unchanged. It is important to note that the reduced frequency, $k=\omega c / v_{c}$, appears only in the vibrating boundaries. If $k \ll 1$ then at the wall $w \simeq 0$, exactly as in a non-vibrating airfoil, but the solid wall boundary location varies with the time. The problem may be seen as a series of independent steady solutions, where the time acts solely as a parameter and the problem is referred to as quasi-steady.

The same problem may be regarded in a different way if we choose as characteristic time $t_{c 2}=\omega^{-1}$, and define the nondimensional time as $\tau_{2}=\omega t$. In this case the non-dimensional time dependent Navier-Stokes equations become: 


$$
k \frac{\partial \tilde{U}}{\partial \tau_{2}}+\tilde{\nabla} \cdot F_{c}(\tilde{U})=\frac{1}{R e} \tilde{\nabla} \cdot F_{v}(\tilde{U})
$$

and the boundary condition for the vibrating wall

$$
\text { at } \tilde{x}=\tilde{x}_{0}+\Delta \tilde{\mathbf{x}} \sin \left(\tau_{2}\right) \quad w=\Delta \tilde{\mathbf{x}} k \cos \left(\tau_{2}\right) .
$$

The reduced frequency appears in this case in the governing equations and in the boundary conditions. It is easy to see that if $k \ll 1$, the boundary condition at the solid wall may be approximated as $w \simeq 0$, exactly as in a non-vibrating airfoil and that the time derivatives may be neglected in first approximation in the governing equations.

The low reduced frequency limit is relevant for aeronautical lowpressure turbines because their slender, high aspect ratio airfoils give rise to low reduced frequency parameters, $k \sim 0.1$.

\section{NUMERICAL MODEL}

\section{Discretised Equations}

The discretised Navier-Stokes equations in conservative form may be written in compact form as:

$$
\frac{\partial U}{\partial t}=R(U)
$$

where $\mathbf{U}$ is the vector of conservative variables. Now we may decompose the flow into two parts: a steady or mean background flow, plus a small but periodic unsteady perturbation, which in turn may be expressed as a Fourier series in time. If we retain just the first harmonic of the series any variable may be expressed as:

$$
\mathbf{U}(\mathbf{x}, t)=\mathbf{U}_{0}(\mathbf{x})+\operatorname{Re}\left(\widehat{\mathbf{u}}(\mathbf{x}) e^{i \omega t}\right)
$$

where $\mathbf{U}_{0}$ represents the background flow and $\widehat{\mathbf{u}}$ is the complex perturbation. The Navier-Stokes equations may then be linearized about the mean flow to obtain:

$$
\left(\frac{d}{d \tau}+i \omega\right) \widehat{\mathbf{u}}=\left(\frac{\partial R}{\partial U}\right)_{0} \widehat{\mathbf{u}}
$$

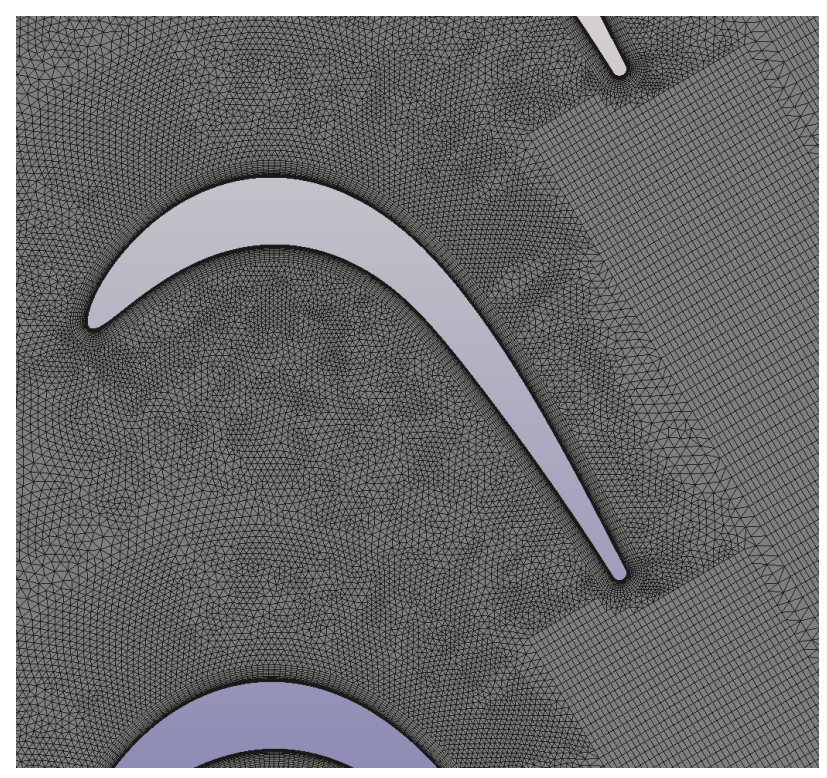

Figure 1. Close-UP OF THE GRID ABOUt A LPT AIRFOIL

which is a system of linear equations with complex coefficients and where the first term is an additional time-derivative added to solve the equations marching in the pseudo-time $\tau$. The linear system of equations is marched in the pseudo time with a RungeKutta and a multigrid technique to accelerate the convergence to the steady state [7]. Turbulent effects are accounted for using the Wilcox 98 turbulence model. The frozen turbulent viscosity approach is followed in the linearised model. The linearity of the flow has been checked previously $[8,9]$ against non-linear simulations and it is shown to be valid if the vibration amplitude, $\delta$, is small enough $(\delta / c \ll 1)$.

A typical low-pressure turbine airfoil operating at design conditions has been chosen to investigate the influence of the reduced frequency and Mach number on the unsteady aerodynamics and the associated work-per-cycle for a flap mode in a direction normal to the engine axis. The inlet and exit angle are respectively, $\alpha_{1}=44^{\circ}$ and $\alpha_{2}=60^{\circ}$, the exit isentropic Mach number is $M_{\text {exit }}=0.75$, the Reynolds number based on the exit velocity $R e \simeq 10^{6}$ to avoid separations in the airfoil suction side and the airfoil spacing, $s / c=0.96$. The space is discretized using an hybrid grid made up of triangles and quadrilaterals, containing about 30,000 nodes per passage and solved using the $M u^{2} s^{2} T$ suite of codes [7, 10]. A close-up of the grid may be seen in Fig. 1.

\section{Airfoil Vibration Approach}

The mode-shape displacements in the airfoils of a perfectly tuned bladed-disk have the same angular frequency, $\omega$, and a common 
inter-blade phase angle, $\sigma$, and may be described as travellingwaves of different wave-lengths. The computational domain may be reduced to a single passage if phase-shifted boundary conditions are used, i.e.: $\hat{u}\left(\theta_{0}+\right.$ pitch $)=\hat{u}\left(\theta_{0}\right) e^{i \sigma}$, which significantly reduces the computational cost but still requires strictly speaking the computation of as many IBPAs as rotor blades to reproduce the whole blade-dynamics.

The force in the $j^{\text {th }}$ airfoil is the sum of the contribution of all the IBPAs

$$
\left\{f_{j}\right\}=\sum_{n} c_{\sigma_{n}} \hat{q}_{\sigma_{n}} e^{i\left(\omega t+\sigma_{n}\right)}
$$

where $c_{\sigma_{n}}$ are the force coefficients of the TWs and $\hat{q}_{\sigma_{n}}$ are the TW coordinates. The same force may be expressed as a function of the displacements of the individual airfoils, $\hat{q}_{j}$, by expanding the TW coordinates as a function of $\hat{q}_{j}$, i.e.: $\left\{\hat{q}_{\sigma_{n}}\right\}=[E]^{-1}\left\{\hat{q}_{j}\right\}$, then

$$
\left\{\hat{f}_{j}\right\}=[L]\left\{\hat{q}_{j}\right\}
$$

where $[L]=[E] \operatorname{diag}\left(c_{\sigma_{1}}, \ldots, c_{\sigma_{N}}\right)[E]^{-1}$ is the so-called influence coefficient matrix and $E_{k, l}=e^{i k \sigma_{l}}=e^{i k \frac{2 \pi l}{N}} . L_{i, j}$ denotes the force in the $i^{t h}$ airfoil due to the displacement of the $j^{\text {th }}$ airfoil. Although matrix $[L]$ is full, it has a very special form and only contains $N$ independent terms due to the cyclic symmetry structure of the problem and the matrix $[E]$. Actually all the elements of the sub-diagonals are identical,

$$
[L]=\left[\begin{array}{cccccc}
L_{0} & L_{-1} & \cdots & L_{-N / 2} & \cdots & L_{1} \\
L_{1} & L_{0} & L_{1} & & \ddots & \vdots \\
\vdots & \ddots & \ddots & \ddots & & L_{-N / 2} \\
L_{N / 2} & & \ddots & \ddots & & \vdots \\
\vdots & \ddots & & \ddots & \ddots & L_{-1} \\
L_{-1} & & L_{N / 2} & & L_{1} & L_{0}
\end{array}\right]
$$

It may also be shown that $L_{p}=\frac{1}{N} \sum_{m=1}^{N} e^{i \frac{2 \pi p}{N} m} c_{m}$, i.e.: $L_{p}$ is the $p^{\text {th }}$ Fourier coefficient of the force coefficients in TW form, i.e.: $L_{p}=\hat{c}_{p}$. Note that $L_{i, j}=L_{p}$ with $p=i-j$. Generally speaking the contribution to the unsteady pressure of the 0 -th airfoil of the airfoils vibrating far away from it is small and therefore the off-diagonal terms located apart form the main diagonal become smaller and smaller. This effectively means that the mean value of the work-per-cycle, $W_{\text {cycle }}$, is due solely to the effect of the airfoil motion in itself. Typically only the adjacent airfoils contribute significantly to the aerodynamic damping $[5,11]$. Fig. 2

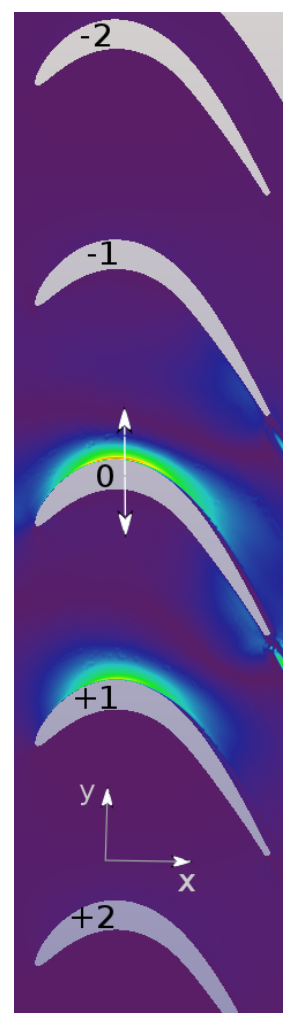

Figure 2. AIRFOIL NUMBERING CONVENTION AND UNSTEADY PRESSURE FIELD CREATED BY THE VIBRATION OF THE 0-TH AIRFOIL

displays the unsteady pressure field generated by the vibration of the central airfoil while the rest are at rest. It may be appreciated that only the 0 -th and +1 th have a significant pressure perturbation while the others remain unperturbed. From a mathematical point of view this means that $L_{0} \neq 0$ and $L_{1} \neq 0$ while the rest of the terms of matrix $[L]$ are negligible in first approximation.

Although from a practical point of view most of the analyses devoted to the computation of aerodamping are performed in TW form injecting the modes extracted from a structural model, from the point of view of physics understanding it is believed that the extraction of the influence coefficients from the simulations and the traversing of the corresponding unsteady fields provides more insight and these should be used in combination with the more widespread simulations in form of TWs.

\section{AERODYNAMIC WORK SCALING}

\section{Unsteady Pressure Modulus}

After many years of research on the unsteady aerodynamics of vibrating airfoils there are a number of phenomenological well established results. From an engineering point of view the most 


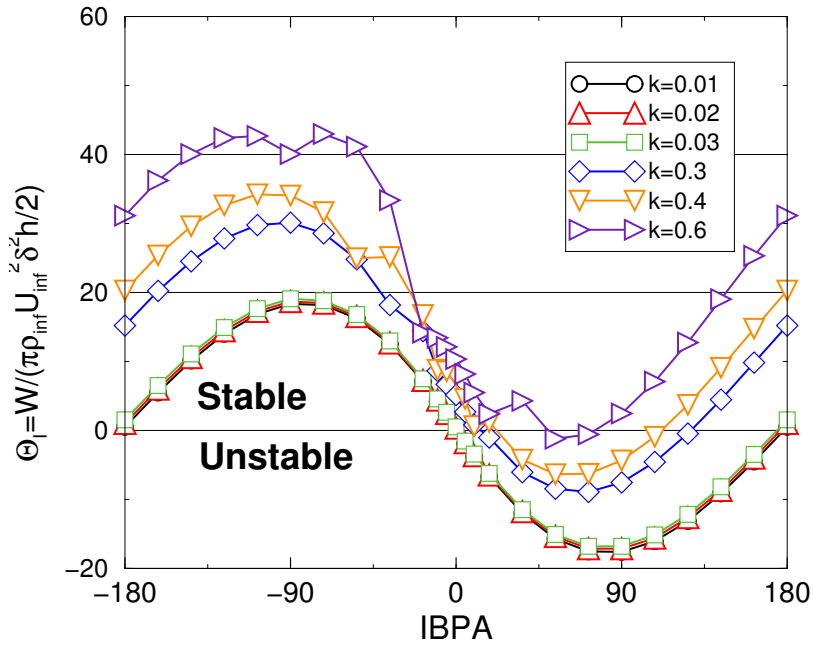

Figure 3. NON-DIMENSIONAL DAMPING (QUASI-UNSTEADY FORM) AS A FUNCTION OF THE IBPA AND THE REDUCED FREQUENCY

relevant and basic result is the stabilization of the damping curves with the reduced frequency. Figure 3 displays the dependence of the non-dimensional work, defined as:

$$
\Theta_{1}=\frac{W_{c y c l e}}{\frac{1}{2} \rho_{e} v_{c}^{2} \delta^{2} h}
$$

of the two-dimensional LPT airfoil that will be studied in this paper with the reduced frequency and the IBPA. The main conclusion that can be drawn is that the minimum damping actually increases with the reduced frequency, as it was previously mentioned. However from a theoretical point of view the nondimensionalization does only a good job at very low reduced frequencies where it is able to collapse several damping curves into a single one. However the stabilization trend with the reduced frequency is not absorbed in the non-dimensionalization. This is due to the fact that scaling the work-per-cycle with $W_{c 1}=\frac{1}{2} \rho_{e} v_{c}^{2} \delta^{2} h$ only makes sense for low reduced frequencies since it completely ignores the dependency with the frequency and assumes that the characteristic pressure is due solely to a quasi-steady motion, i.e.: the characteristic unsteady pressure is a fraction of the dynamic pressure, $p_{c 1}=\frac{1}{2} \rho_{e} v_{c}^{2}(\delta / c)$.

The second remarkable result is that damping curves may be approximated by a mean value plus a sine-like dependence with the IBPA, this effectively means that solely the \pm 1 adjacent blades are actively involved in the dynamics $[5,11]$. Also the shape of the curves signals a non-symmetric behaviour of the two adjacent blades as it is shown in Figure 2. This is due to both, the low reduced frequency and the higher velocity of the SS of the

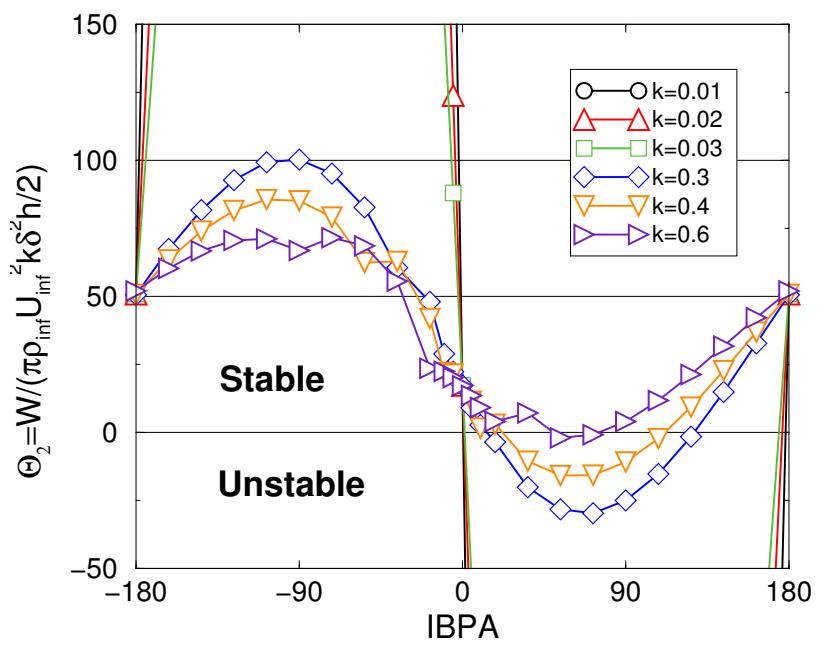

Figure 4. NON-DIMENSIONAL DAMPING (UNSTEADY FORM) AS A FUNCTION OF THE IBPA AND THE REDUCED FREQUENCY

+1 airfoil than that of the PS of -1 airfoil that it is ultimately responsible of the different sensitivity of the adjeacent airfoils to the vibration of the central one. It will be clearly shown later on that this asymmetry in the response of the \pm 1 airfoils is ultimately responsible for the minimum work-per-cylce occurs at $\sigma=90^{\circ}$. This behaviour may be seen in any lifting cascade of airfoils vibrating at low reduced frequencies. It may be appreciated that at very low reduced frequencies the system is always aerodynamically unstable and in practice the system may be only stabilised by recurring to mechanical friction.

Alternatively, work-per-cycle may be non-dimensionalised assuming that the unsteady pressure is due to the velocity induced by the airfoil vibration and not by the change in the nominal position. In this case the characteristic pressure scales as $p_{c 2}=\frac{1}{2} \rho_{e} v_{c}(\omega \delta)$ and therefore $p_{c 2}=k \cdot p_{c 1}$. The corresponding non-dimensional work-per-cycle is then

$$
\Theta_{2}=\frac{W_{c y c l e}}{\frac{1}{2} \rho_{e} v_{c}^{2} k \delta^{2} h}
$$

The non-dimensional work-per-cycle using the above nondimensionalization is displayed in Fig. 4. It may be seen that the curves corresponding to very low-reduced frequencies are out of range and are not properly scaled since in the limit $k \rightarrow 0$, $\Theta_{2} \rightarrow \infty$, as it was expected. However it is interesting to note that non-dimensional work for $\sigma=180^{\circ}$, which is a very good indicator of the mean non-dimensional work in this particular case, collapse to a single value for the whole range of reduced frequencies. On the contrary that it could be thought this does not 


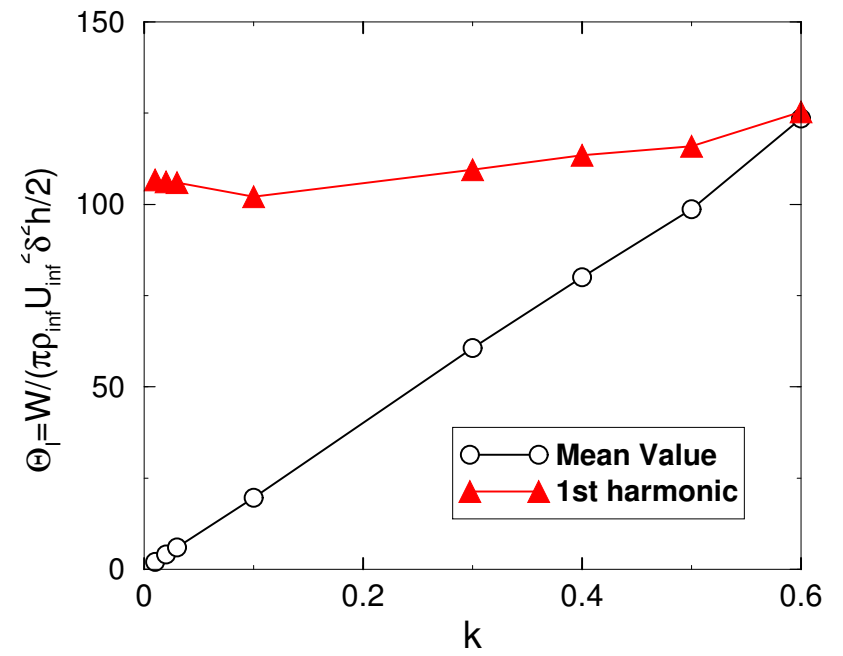

Figure 5. HARMONICS OF THE $1^{\text {st }}$ FORM OF THE NONDIMENSIONAL WORK AS A FUNCTION OF THE REDUCED FREQUENCY

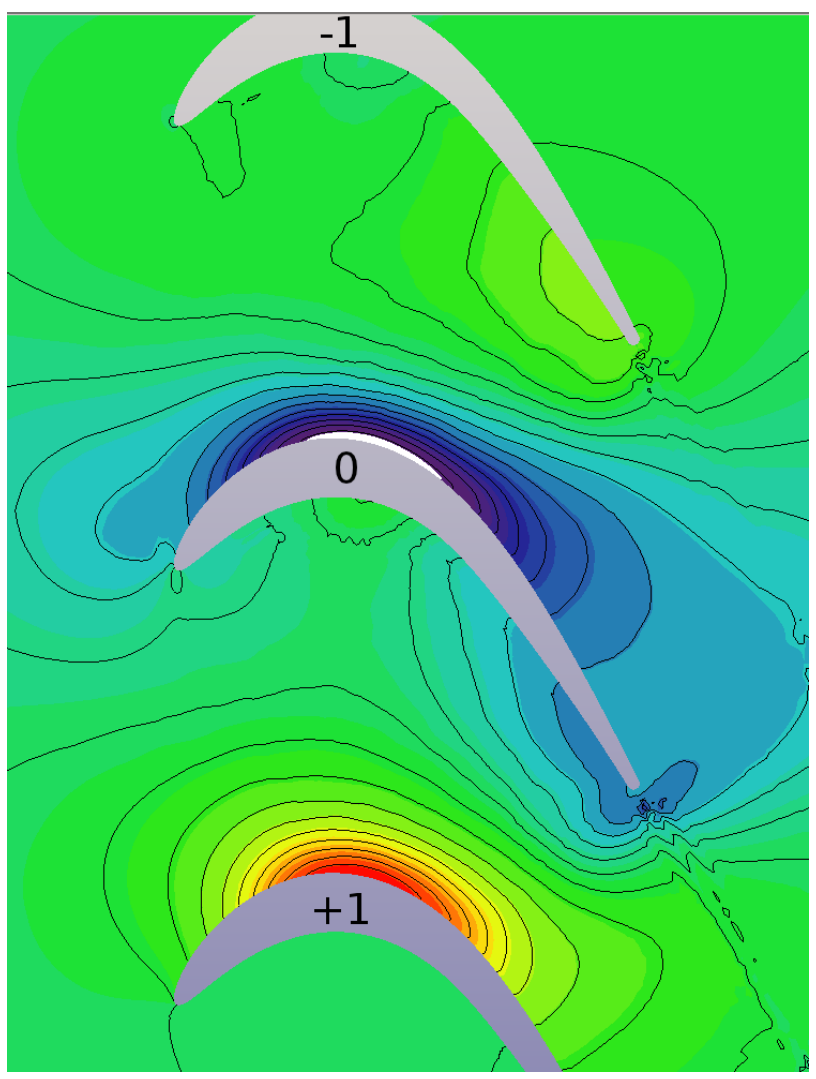

Figure 6. SNAPSHOT OF THE UNSTEADY PRESSURE CAUSE BY THE VIBRATION OF THE CENTRAL BLADE IN A LPT AIRFOIL FOR $k=0.3$

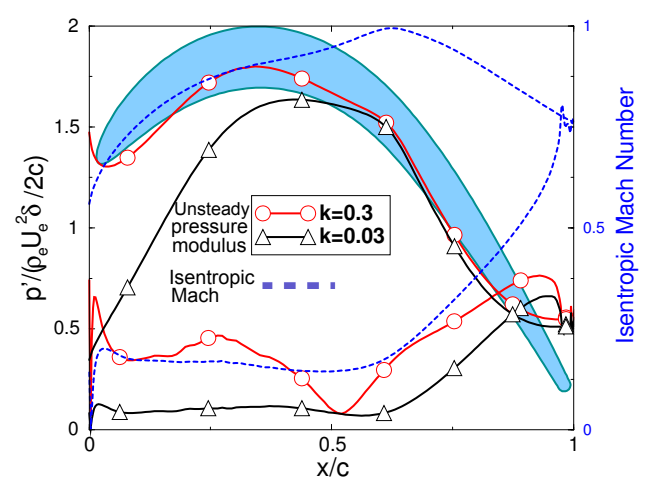

Figure 7. ISENTROPIC MACH NUMBER AND UNSTEADY PRESSURE MODULUS DISTRIBUTIONS ALONG BLADE SURFACE FOR THE 0TH AIRFOIL

actually mean that the unsteady pressure of +0 cascade airfoil, i.e., the one that causes the perturbation it is dominated by the unsteadiness caused by the velocity induced by the airfoil vibration and that adjacent blades have a different physics and scale in a completely different manner with the reduced frequency. This behaviour is, as it will be shown later on, due to the special way that airfoil phasing contributes to $W_{\text {cycle }}$.

Figure 5 shows the amplitude of the mean and $1^{s t}$ harmonic of the $1^{\text {st }}$ form of the non-dimensional work as a function of the reduced frequency. It is clearly seen that while the mean value of the non-dimensional work scales linearly with the reduced frequency the $1^{s t}$ harmonic is fairly unaffected by the frequency. This is the ultimate reason why a global scaling of the nondimensional work curves does not work since the scaling has to be performed in an harmonic by harmonic basis.

A simple model for the non-dimensional work-per-cycle as a function of the IBPA for the reduced frequency range of interest for a given mode-shape would then be

$$
\Theta_{1}=\Theta_{1,0} k+\Theta_{1,1} \sin (\sigma+\phi(k)),
$$

where it may be seen that the stabilization associated to an increase of the reduced frequency is mainly due the the central blade, while the first harmonic is a quasi steady characteristic that depends on the steady field. A frequency increase gives rise also to a shift in the damping curves that may be seen both in Figs. 3 and 4 and modifies the IBPA at which the minimum damping occurs.

\section{Physical Interpretation I}

It is convenient to interpret the aforementioned results in physical terms. Figure 6 displays a snapshot of the unsteady pressure of 

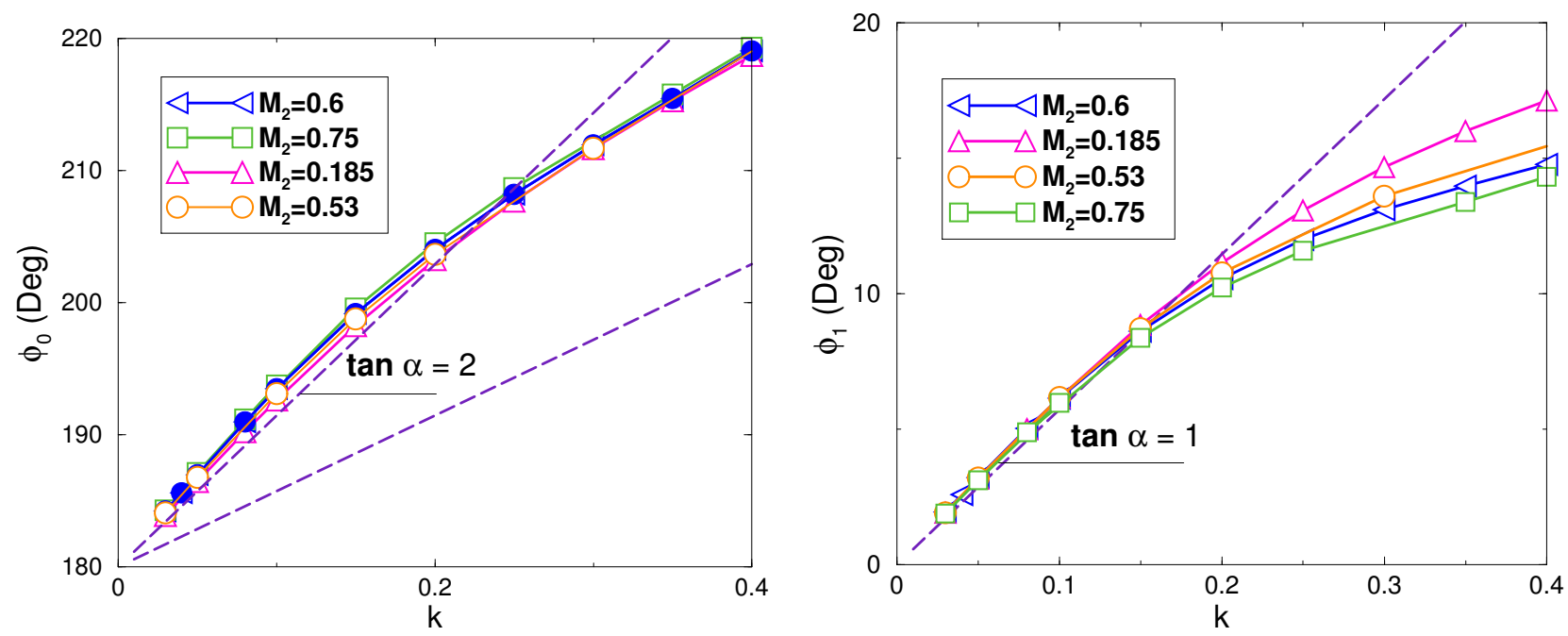

Figure 9. Phase of THE INFLUENCE COEFFICIENTS OF THE 0TH (LEFT) AND +1St (RIGHT) AIRFOILS AS A FUNCTION OF THE STROUHAL AND MACH NUMBERS.

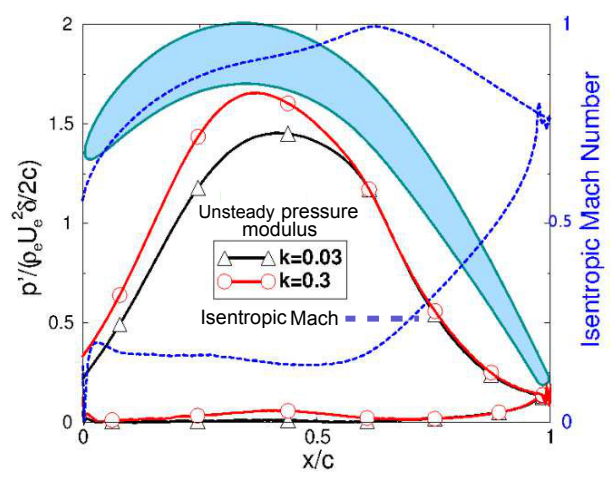

Figure 8. ISENTROPIC MACH NUMBER AND UNSTEADY PRESSURE MODULUS DISTRIBUTIONS ALONG BLADE SURFACE FOR +1 AIRFOIL 1

a cascade of 5 airfoils where only the $0^{\text {th }}$ airfoil moves in bending motion at $S t=0.3$. It may be appreciated first that only the $0^{t h}$ and the $+1^{s t}$ airfoils are perturbed by the motion of the central blade and that the highest unsteady pressure is located in the region of highest Mach numbers, as it can be seen in figures 7 and 8 which represent the Isentropic Mach number and the unsteady pressure distributions along blade surface of blade 0 and +1 respectively. At this point it is important to highlight that:

1. Only the suction side of the 0 and +1 airfoils are affected by the perturbation. This is a purely quasi-steady effect since the peak Mach number is the profile's location with the highest sensitivity to variations in the airfoil location (Fig. 7 and

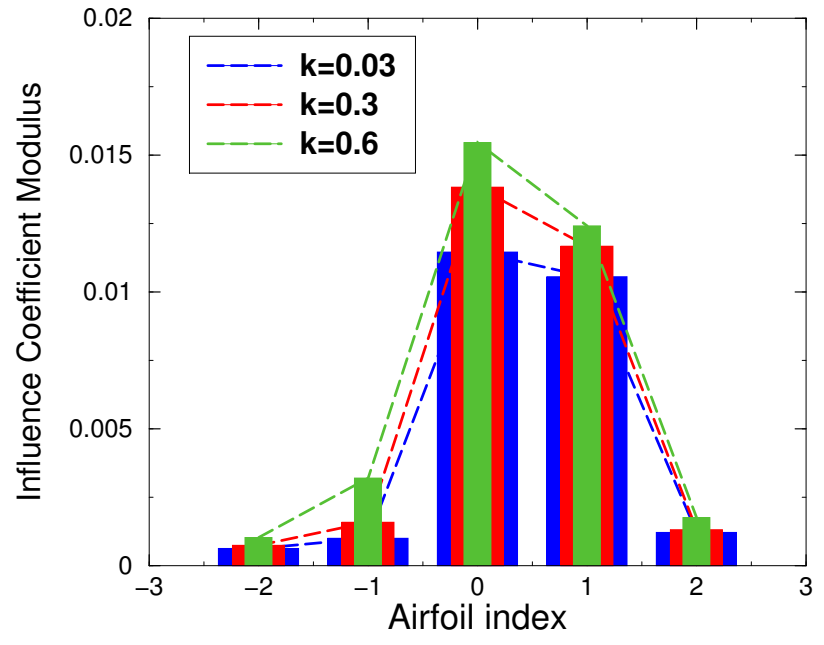

Figure 10. MODUluS OF THE FORCE INFLUENCE COEFFICIENTS AS A FUNCTION OF THE REDUCED FREQUENCY FOR $M_{2}=0.75$

8)

2. The unsteady pressure in the suction side of the airfoil +1 is not the result of an acoustic perturbation originated in the pressure side of the $0^{\text {th }}$ airfoil since actually the unsteady pressure in the pressure side of this airfoil is negligible. There are no tracks in Fig. 6 of perturbations generated in the PS of the central blade reaching the SS of the neighbouring airfoil. Variations in the static pressure are caused in this case by the pitch variation due to the bending of the central airfoil. The wave-length of an acoustic 
perturtbation originated by the vibrating airfoil, $\lambda$, would be $\lambda=T . a$, where $a$ is the speed of sound and $T$ the vibration period, then $\lambda / c=2 \pi(a / \omega c)=2 \pi /(M . S t)$, if $\mathrm{M}=0.6$ and $\mathrm{St}=0.3$, then $\lambda / c \simeq 35$. This wave-length does not fit neither in the computational domain nor in any linear vibrating cascade, and may not be seen in the contour plots, actually the wave-length of the perturbations in the pitch-wise direction is much shorter. This is a clear indication that acoustic waves play a secondary role in this type of flows.

3. The SS of the central and the +1 airfoil are in first approximation in anti-phase. When the central blade moves downwards (as it happens in the time instant displayed in Fig. 6) the loading of the SS increases and therefore the static pressure decreases. The opposite is true for the SS of the airfoil -1 .

4. The basic idea is that the flapping motion of the central airfoil in the $y$-direction does not change in first approximation the exit angle of the adjacent passages and therefore the total lift of the cascade remains constant. This effectively means that in a quasi-steady motion if the lift of one of the airfoils increases the lift of the rest of the airfoils must decrease accordingly in order that the global lift remain constant. In this particular mode the balance is obtained exchanging the lift of the central and +1 airfoil.

All these observations are aligned with the behaviour previously described for the work-per-cycle as a function of the reduced frequency and the different scaling of the mean and first harmonic with the frequency. However to obtain the whole picture it is necessary to inspect the phasing of the airfoils.

\section{Phasing Variation}

The phase of the unsteady pressure on the central and +1 airfoils with respect the airfoil displacement, $\phi$, plays a central role in the overall damping of the system and therefore it has been inspected in great detail. Figure 9 shows the dependence of the influence coefficient phase of the central, $\phi_{0}$, and $+1, \phi_{+1}$, airfoils as a function of the reduced frequency and Mach number. It may be seen that both, in a large range of the reduced frequency, scale linearly. It is interesting to observe that $\phi_{0} \simeq 2 k$ while $\phi_{1} \simeq k$ and that this seems to be a general trend, actually the sensitivity to the Mach number is fairly low.

This is consistent with the variation of the mean value and 1st harmonic of the reduced frequency displayed in Fig. 5. If we assume that we may neglect the influence of all the airfoils, except that of the central and adjacent airfoils in the work per cycle (see [5] and Fig. 10) then it may be shown that

$$
W_{\text {cycle }}=-\pi q_{0}^{2}\left[l_{0} \sin \phi_{0}+l_{+1} \sin \left(\phi_{1}+\sigma\right)+l_{-1} \sin \left(\phi_{-1}-\sigma\right)\right]
$$

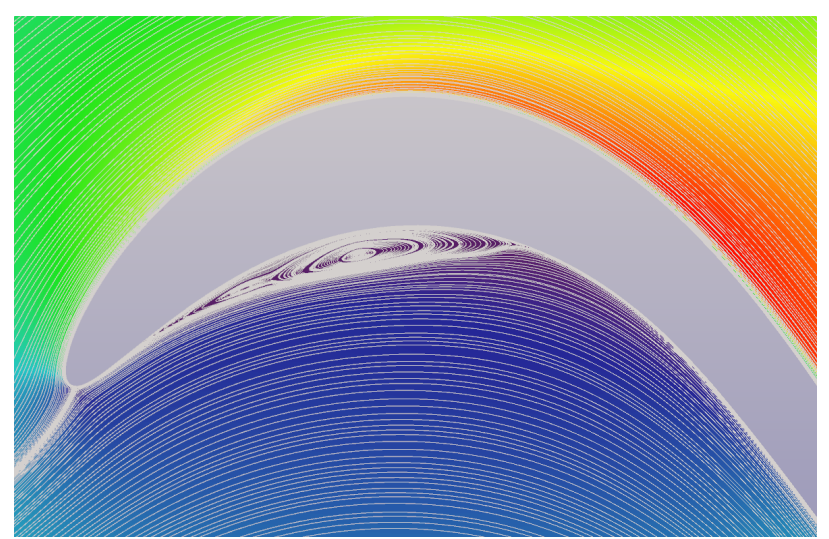

Figure 12. MACH NUMBER ISO-CONTOURS AND STREAMLINES

where $l_{j}$ and $\phi_{j}$ are respectively the modulus and phase of the $j^{t h}$ influence coefficient and $q_{0}$ the modulus of the vibration amplitude. Moreover if the reduced frequency is small enough, and therefore $\phi_{j} \ll 1$, and if $l_{-1} \ll l_{+1}$, then the expression 15 , neglecting second order terms, reduces to:

$$
W_{\text {cycle }}=-\pi q_{0}^{2} l_{+1}\left[\left(\frac{l_{0}}{l_{+1}}\right) \phi_{0}+\sin \left(\phi_{1}+\sigma\right)\right],
$$

the phase of the 0th influence coefficient is responsible of the damping increase with $k$, which in first approximation is linear since $\phi_{0} \simeq 2 k$. This is consistent with Figs. 3 and 5. The phase of the +1 airfoil is responsible of the shift of the minimum damping location. The small values of the phase associated to the low reduced frequency regime are ultimate reason of the sinusoidal shape of the work-per-cycle as a fuction of the IBPA curve. Moreover if the contribution of the -1 airfoil is much smaller than that of the +1 airfoil the minimum of the curve takes place at $\sigma \simeq 90^{\circ}$. Note that this approximation breaks approximately for $k \simeq 0.2$ when the linearity of the phases with $k$ does not hold anymore.

\section{Physical Interpretation II}

Phase behaviour reveals a number of interesting physical phenomena. At very low reduced frequencies $(k \ll 1)$ the phase tends either to zero or $180^{\circ}$. This is consistent with the quasisteady approach in which the perturbations are either in phase or anti-phase, i.e.: pressure increases or decreases with the motion instantaneously. It is important to recall that the reduced frequency

$$
k=\frac{c / v_{c}}{\omega^{-1}}=\frac{t_{\text {residence }}}{t_{\text {characteristic }}}
$$



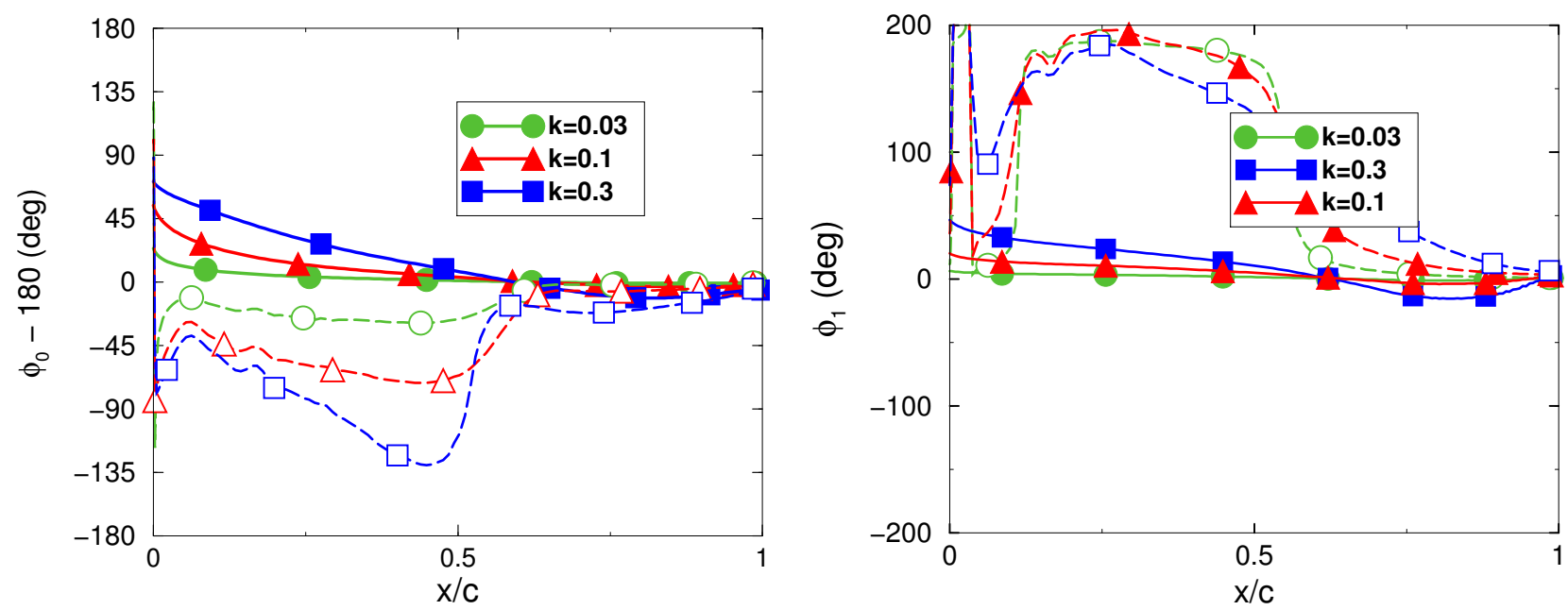

Figure 11. PHASE Distribution ALONG THE 0-TH (LEFT) AND + 1-TH (RIGHT) AIRFOILS FOR DIFFERENT REDUCED FREQUENCIES. SOlID LINE: SuCTION SIDE. DASHED LiNE: PRESSURE SIDE

is the ratio between the time of residence, $t_{r}=c / v_{c}$, and the characteristic time, $\omega^{-1}$. When $k \ll 1$ fluid particles traverse the domain as many as $k^{-1}$ times during a vibration cycle and therefore the flow is quasi-steady and $\phi=0$ along the whole airfoil (see Fig. 11). When the frequency is increased, at it may be appreciated in the same figure, the phases of both, the airfoil 0 and +1 , vary nearly linearly along the chord. This effectively means that there is a travelling wave moving from the leading to the trailing edge of the airfoil. Let us assume that $\Delta p=p^{\prime}(x) e^{i(w t+\phi)}$ and that $\phi=\phi_{L . E .}(1-x / c)$ then the velocity at which the pressure perturbations propagate is, $v_{p}=\omega c / \phi_{L: E}$. If we non-dimensionalise $v_{p}$ using the characteristic velocity $v_{c}$, then $v_{p} / v_{c}=k / \phi_{L . E .}$. The fact that the airfoil's phase scale linearly with the reduced frequency is a direct consequence that the velocity of the perturbation scales with the convection velocity $v_{c}$. Comparing the phases of the central and +1 airfoils it may be concluded that the events in the central airfoil take place first in time since this is the blade that is actually moving. The phase difference between the leading and the trailing -edge of either the central or the +1 airfoil for the case $k=0.3$ is about $60^{\circ}$ (see Fig. 11). This means that the the residence time of the perturbations running downstream of the the airfoil is $t_{\text {residence }} / T \simeq 1 / 6$, taking into account that $t_{\text {residence }} / T=(1 / 2 \pi)\left(c_{\text {true }} / c\right) k \simeq 1 / 12$, where we have assumed that the ratio between the true and the axial chord, $c_{\text {true }} / c$, is roughly 1.5. Both estimates are consistent and show that the velocity of propagation is of the order of the convection velocity. The variation in the circulation due to the airfoil's motion is adjusted by vortex shedding that ultimately is due to vorticity convection along the airfoil chord according with the vorticity equation. It is concluded that all the evidences point out that unsteadiness is caused by an adjustment of the circulation of the central airfoil whose response time is of the order of the chord

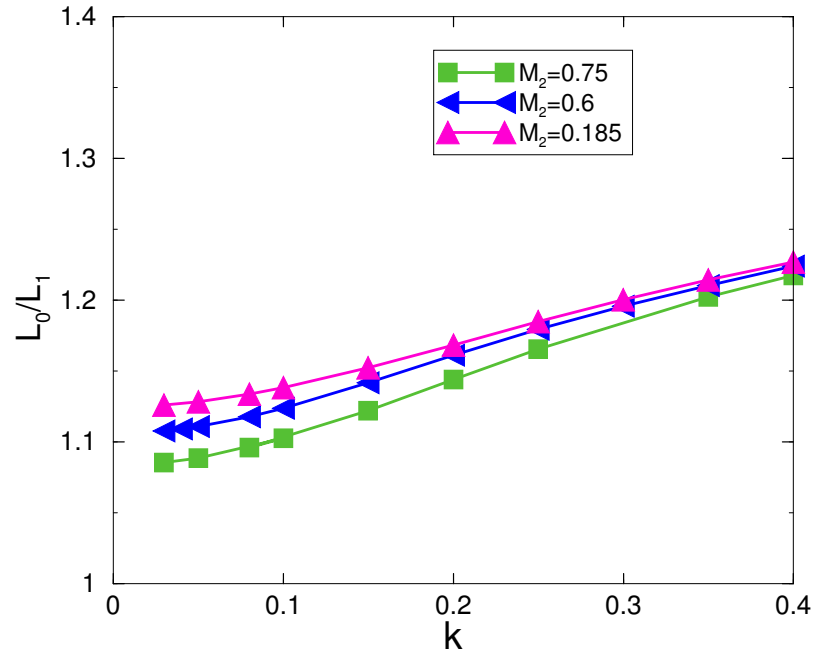

Figure 13. RATIO BETWEEN THE MODULUS OF INFLUENCE COEFFICIENT 0 AND + 1 AS A FUNCTION OF THE REDUCED FREQUENCY AND MACH NUMBER

divided by the mean convection velocity.

Pressure side phase exhibits a more complex behaviour (see Fig. 11 , dashed lines), in general the phase variation along the airfoil is much steeper than in the suction side. Following the previous argument, this is a direct consequence of the fact that the propagation velocity, which is associated to the mean convection velocity, is significantly lower in the separated region of the PS than in the SS, as it may be appreciated in Fig.12, this causes an abrupt change in the phase in the reattachment point (see Fig. 11 , left) around $x / c \simeq 0.5$. The phase of the PS of +1 follows a 
similar behaviour than that of the 0th airfoil with steep variations of the phase caused by low momentum separated flow.

\section{WORK-PER-CYCLE MODEL}

Considering all the previous results it is easy to derive a simple model for the work-per-cycle of this mode based in the expression 16, that relates the work-per-cycle with the influence coefficients. Since it have already been stated that $\phi_{0} \simeq 2 k$ and $\phi_{1} \simeq k$, to close the model an expression for the ratio between the influence coefficients, $l_{0} / l_{+1}$ and for $l_{+1}$ is needed. The ratio has a direct impact in the determination of the stability region, the critical reduced frequency, while the actual value of the IC affects only the severity of the instability.

Figure 13 represents the $r=l_{0} / l_{+1}$ ratio as a function of the reduced frequency and Mach number. It may be observed that in the range of interest this ratio is fairly constant, increasing slightly with the reduced frequency, about $10 \%$ when $k$ is increased from 0 to 0.4 . The variation with the Mach number is smooth as well, the ratio drops about $10 \%$ when $M_{\text {exit }}$ is changed from 0.2 to 0.75 . Hence we may model the ratio as $r=r_{0}+\alpha k$ with $r_{0} \simeq 1.1$ and $\alpha \simeq 0.2$. For a flapping mode the exit angle of all the airfoils of the cascade is the same and then the total lift generated by them must be constant in a quasi-steady regime. The lift increase generated in the central airfoil when it moves downwards and the pitch of the suction side passage is increased has to be balanced by the lift decrease of the +1 airfoil that sees a decrease of the pitch of its suction side passage. Therefore $l_{0} \simeq l_{+1}$ in first approximation irrespectively of the reduced frequency and Mach number. The missing lift (around 10\% in this case) is compensated by the contribution of the rest of the airfoils.

The critical reduced frequency, $k_{c}$, may be derived from

$$
\left(r_{0}+\alpha k_{c}\right) 2 k_{c}-1=0
$$

The most important conclusion is that the stability limit is weakly dependent on the Mach number.

Figure 14 shows that the dimensional IC of the 0th airfoil, $l_{0}$, scales quadratically with the Mach number while the dependence with the reduced frequency is much weaker. The effect of the Mach number is then in first approximation to increase the severity of the instability but not the stability limit.

These two facts may be verified deriving directly the damping as a function of the IBPA for different Mach numbers. Figure 15 represents the variation with the Mach number of the $1^{\text {st }}$ form of the non-dimensional work, obtained by the TW formulation. It

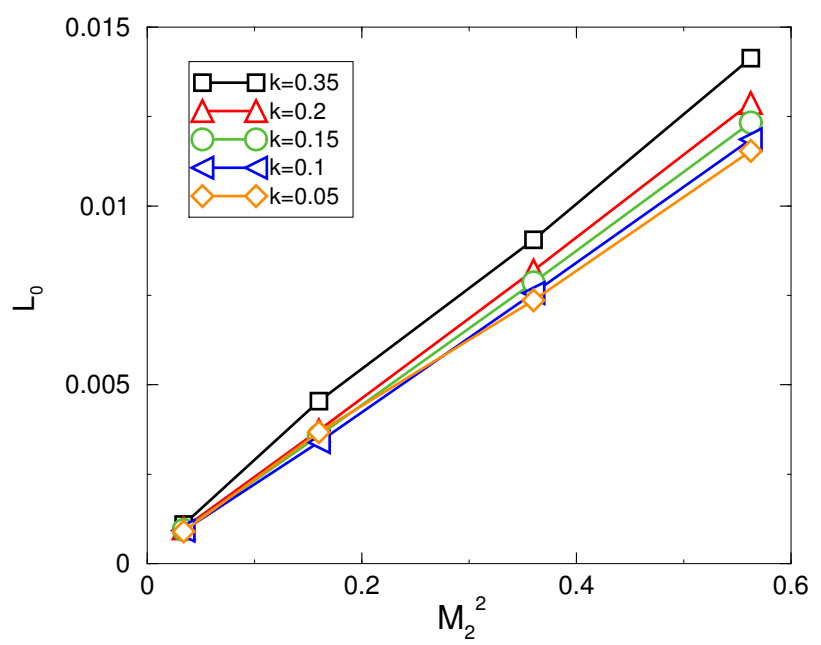

Figure 14. VARIATION OF THE INFLUENCE COEFFICIENT OF THE CENTRAL BLADE WITH THE SQUARE OF THE MACH NUMBER AND THE REDUCED FREQUENCY

may be observed that while the effect of changing only the reduced frequency but not the Mach number is a shift in the damping curve and an increase in the mean damping, the Mach number effect is in first approximation very small, much less than that of the dimensional influence coefficient displayed in Fig. 14. This is due to the fact that the effect of the Mach in the dynamic pressure has already been retained in the non-dimensionalisation while the effect of the acoustics has been shown to be small and therefore all the curves tend to coalesce in a single one, especially in the minimum damping region. Damping curves for different Mach still exhibit a small scaling effect but the stability region is fairly unaffected, as it was claimed before. Figure 16 shows the amplitude of the mean and $1^{s t}$ harmonic of the $1^{s t}$ form of the non-dimensional work as a function of the square of the Mach number. It can be still seen that both scale weakly with Mach number square.

Note that since $k \ll 1$ and $\alpha \simeq 0.2 \Rightarrow \alpha k \ll r_{0} \Rightarrow r \simeq r_{0}$ a model for the work per cycle for this mode can be written according to the following formula:

$$
W_{\text {cycle }}=-\pi \beta q_{0}^{2} M^{2}\left[2 r_{0} k+\sin (k+\sigma)\right] .
$$

This is a comprehensive model for $W_{\text {cycle }}$ that includes the influence of the Mach and the reduced frequency. 


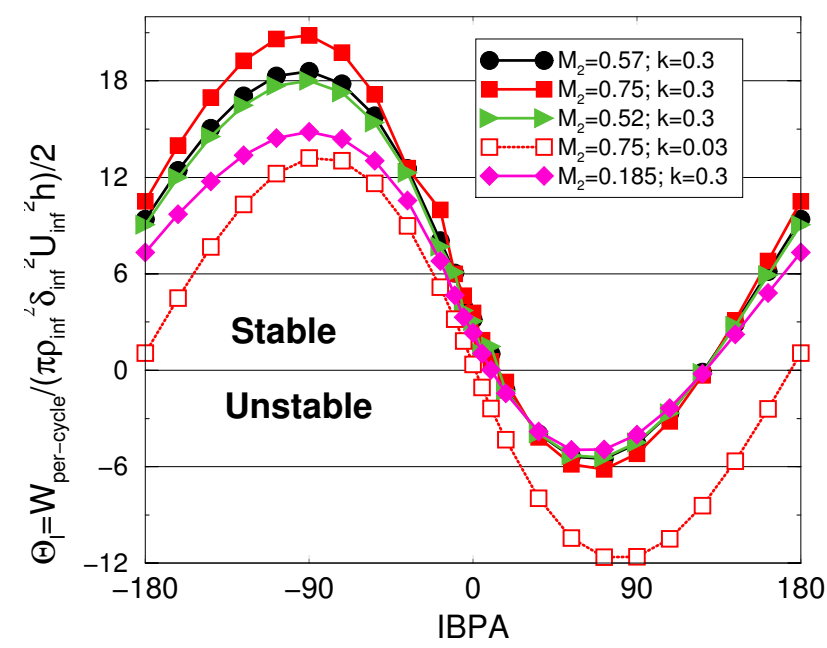

Figure 15. NON-DIMENSIONAL DAMPING (QUASI-UNSTEADY FORM) AS A FUNCTION OF THE IBPA AND THE MACH NUMBER

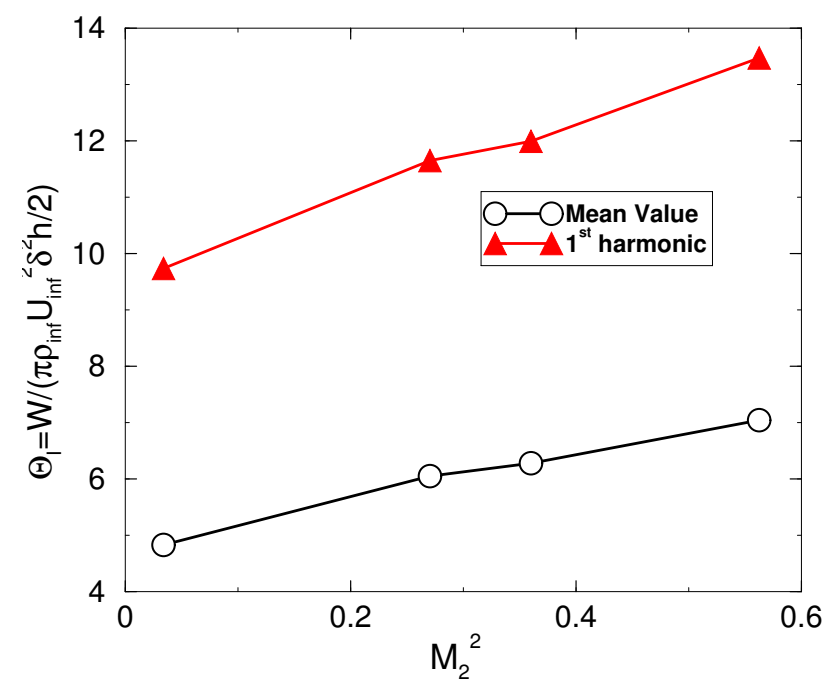

Figure 16. HARMONICS OF THE $1^{\text {st }}$ FORM OF THE NONDIMENSIONAL WORK AS A FUNCTION OF THE MACH NUMBER

\section{MODE-SHAPE EFFECTS}

It is well known that the sensitivity of the unsteady aerodynamics of vibrating airfoils to the mode-shape is very high $[12,1]$. The objective of this section is to understand how general are the results presented in the previous sections for the flap mode. Two more modes have been investigated, the edge-wise mode, represented as a vibration in the $\mathrm{x}$-direction, and a torsion mode about a point located in the airfoil's meanline at the maximum thickness location. The same parametric study that was conducted for the flap mode has been performed for both modes. For the sake of brevity just a reduced set of data will be presented here.

\section{Edge-wise Mode}

Some of the conclusions that were drawn for the flap mode apply also to the edge-wise mode. The non-dimensional work as a function of the IBPA and the reduced frequency may be seen in Fig. 18 (Top). It may be appreciated that at low-reduced frequencies the damping curves have a sine-like shape, which is a direct indication that only the airfoils adjacent to the central contribute to the work-per-cycle, however, on the contrary than in the flap mode both adjacent airfoils are equally important. The trend of the mean value and the first harmonic of the work-per-cycle with the reduced frequency is the same than for the flap mode and therefore they require also the same differential scaling than the flap mode. The influence coefficients scale quadratically with the Mach number and the ratios $l_{+1} / l_{0}$ and $l_{+1} / l_{-1} \simeq 1$ are fairly constant with the Mach number and the reduced frequency, even in a wider range than for the flap mode. Figure 17 displays the phases of the 0th and \pm 1 airfoils. It may be seen that the phases of all the airfoils vary linearly with the reduced frequency and the dependence with the Mach number is very weak. The edgewise mode exhibits some differences with the flap mode. While the only two significant airfoils from the point of view of the size of the pressure perturbation in the flap mode, the 0th and the +1 airfoils, are dominated by vorticity waves and are therefore fairly insensitive to Mach number variations, the 0th and -1 airfoils of the edge-wise mode show some sensitivity to the Mach number. This presence of acoustic waves helps to explain why the suction side of the 0th and the pressure side of the - 1 airfoil are in antiphase for any Mach number and reduced frequency. This is only possible if the effective velocity of propagation, the sound speed, is much larger and therefore both surfaces are synchronized at any instant and flow condition. Vorticity waves, superimposed with the aforementioned acoustic waves, may also be seen in the 0th airfoil, however the precise contribution of each of them cannot be done without a proper mode decomposition. The influence of acoustic waves in the edge-wise mode may be appreciated also in work-per-cycle (Fig. 18 Top) where the spikes associated to the inlet and outlet resonance conditions may be seen. This is only possible if the pressure perturbations associated to the acoustic waves are at least of the same order than those associated to the vorticity waves. Note that under the same conditions these spikes are not visible in the flap mode, that is solely controlled by vortex shedding mechanisms. Moreover the variation of the non-dimensional aerodynamic work-per-cycle (Not shown here for the sake of brevity) displays a large dependence with the Mach number. Another significant difference is that although the modulus of the unsteady pressure of the -1 airfoil is small, its contribution to the work-per-cycle is comparable to that of the +1 airfoil. Plotsfor other variables exhibit an equivalent degree 


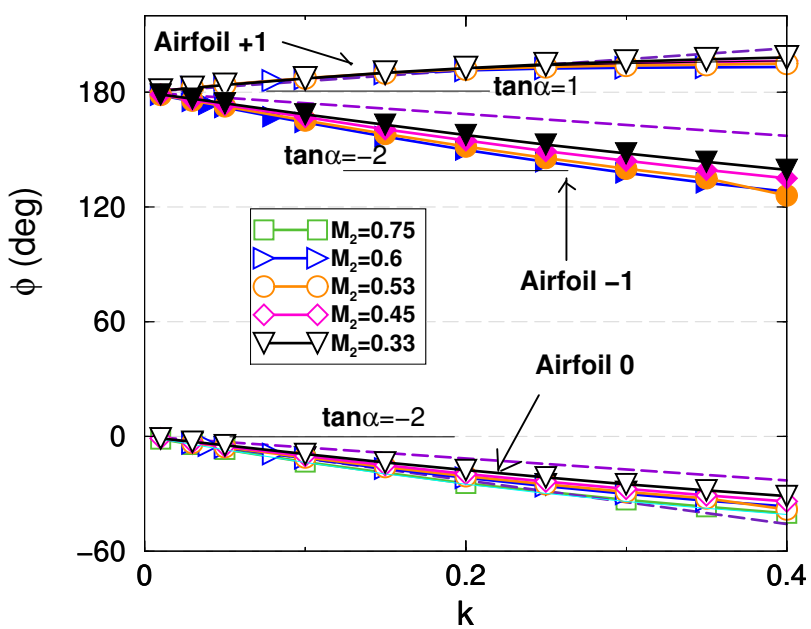

Figure 17. PHASES OF THE 0TH AND \pm 1 AIRFOILS AS A FUNCTION OF THE REDUCED FREQUENCY AND MACH NUMBER OF

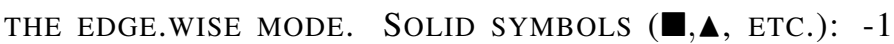
AIRFOIL, Filled SYMBOLS $(\square, \triangle$, ETC.): 0TH AND + 1 AIRFOILS.

of similarity with the flap mode. Summarising a similar model retaining the $k$ and Mach number dependence than that obtained for the flap mode could be derived although a special treatment of the Mach number effect would be required,

\section{Torsion Mode}

The torsion mode is also more difficult to interpret than the flap mode. We will concentrate here in describing just the main differences that may be summarised in the following two observations. The first is that the contribution to the work-per-cycle of the \pm 2 airfoils is relevant. This may be concluded just by realizing that the work-per-cycle as a function of the IBPA is a discontinuous saw-tooth function (see Fig. 18 , bottom) with a rich harmonic content. Figure 19 displays the moment influence coefficients and it may be appreciated that the contribution of airfoils far away from the central one is still significant. It may be seen (see Fig. 20) that the modulus of the unsteady pressure in the 0th and the -1 airfoil is much larger than that of the rest of the airfoils. However due to torsion nature of the motion the unsteady pressure contribution to the moment of the front and rear part of the airfoil cancel out given rise to a relatively low contribution of these airfoils. On the contrary, the -2 . -3 and 4 have a relatively small unsteady pressure in the trailing-edge region that is amplified when the moment with respect the central part of the airfoil is computed. The same is true for the +2 , +3 and +4 airfoils but with the leading-edge region. The torsion mode motion, on the contrary than the flap and edge-wise modes, changes the exit angle, and therefore the lift, of the cascade. The system reacts creating upstream running pressure waves departing from the domain exit that can be clearly seen in the simulations. When the exit angle of the central airfoil is changed the whole cascade reacts adjusting the mass-flow. This information is transmited upstream by means of pressure waves. It may be seen in Fig. 21how the phase of the central airfoil is independent of the Mach number, this means that the unsteady pressure is controlled by vortex shedding. However the phases of rest of the airfoils, in anti-phase with the central one, depend with the Mach number, which a clear indication that they are dominated by acoustic waves.

The second difference is that the Mach number sensitivity of the \pm 2 and +1 airfoil phases is noticeable and therefore the sensitivity of the non-dimensional work-per-cycle to the Mach number is higher than for the flap mode but lower than for the edge mode. The Mach number sensitivity of the airfoils located far away from the central, and that are dominated by the acoustic waves, is a general trend for all the modes. Moreover the reduced frequency range for which the phase trend is linear is narrower. As a consequence the work-per-cycle model described in Eq. 16 needs to be extended to include more ICs and to take into account Mach number effects.

\section{CONCLUDING REMARKS}

The unsteady aerodynamics of a typical LPT airfoil vibrating in bending and operating at high subsonic conditions has been numerically studied. The edge-wise and torsion modes have been briefly described because of the sake of brevity, but a complete study has been performed and will be published in the future. The effect of the unsteady aerodynamics on the aeroelastic stability of the airfoil has been investigated using both the TW and IC formulation. The main findings of the present investigation can be summarised in the following way:

1. The scaling of the work per cycle needs to take into account the different trends with the reduced frequency of the mean damping and the variations with the IBPA.

2. The phase between the unsteady pressure and the airfoil displacement scales linearly with the reduced frequency.

3. The unsteady pressure in the flap mode is caused mainly by convective vorticity modes in the central and adjeacent airfoils. Acoustic perturbations does not play any role in the low reduced frequency regime. This is supported by the fact that the phases of the influence coefficients are fairly independent of the Mach number although their modulus may depend with the Mach.

4. The effect of the Mach number in the flap mode is a pure scaling of the damping curves and therefore it does not change the stability region but the severity of the instability, that increases with the Mach number 

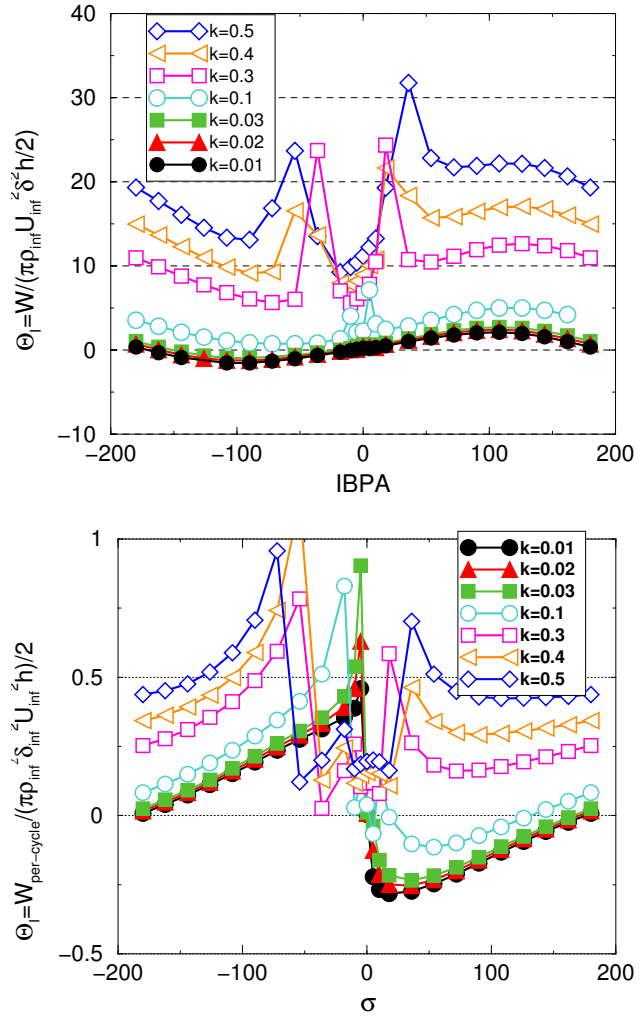

Figure 18. NON-DIMENSIONAL DAMPING (QUASI-UNSTEADY FORM) AS A FUNCTION OF THE IBPA AND THE REDUCED FREQUENCY FOR THE EDGE-WISE (TOP) AND TORSION (BOTTOM) MODES

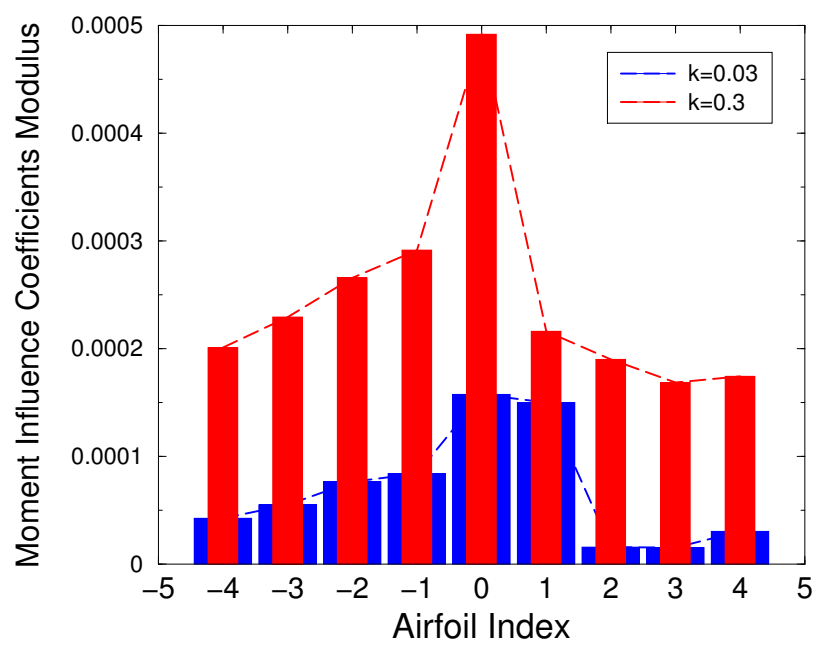

Figure 19. MOMENT INFLUENCE COEFFICIENTS OF THE TORSION MODE AT TWO DIFFERENT REDUCED FREQUENCIES AND $M_{2}=0.75$

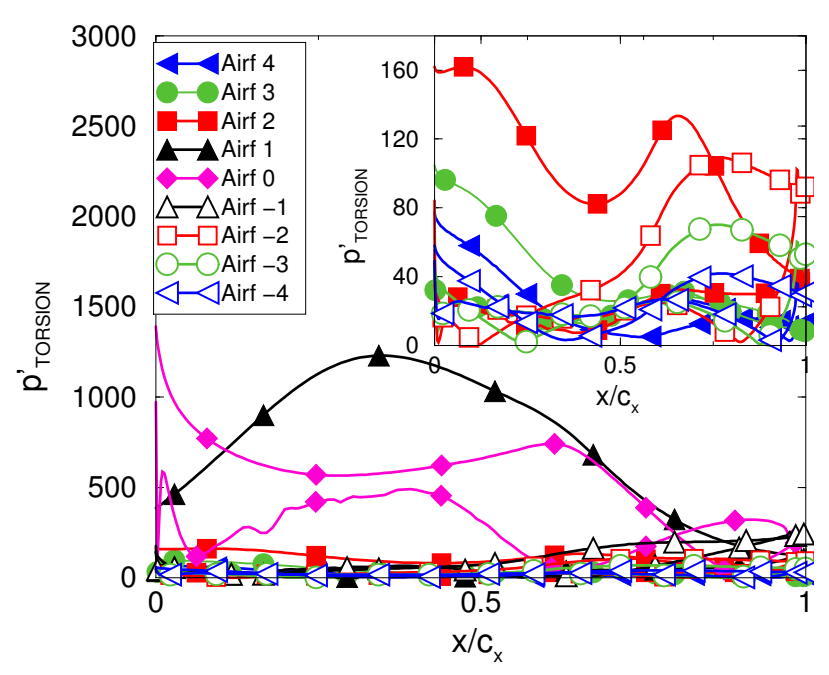

Figure 20. MODUluS OF THE UNSTEADY PRESSURE DISTRIBUTION ALONG THE AIRFOILS OF A LINEAR CASCADE COMPOSED OF NINE AIRFOILS $\left(M_{2}=0.75\right)$

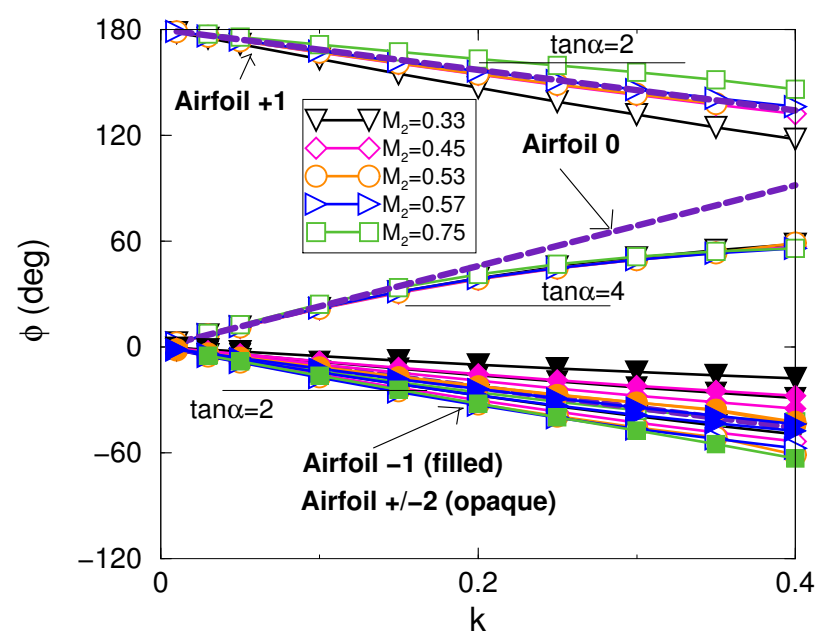

Figure 21. PHASES OF THE INFLUENCE COEFFICIENTS OF THE TORSION MODE FOR DIFFERENT MACH NUMBERS

5. Not all the conclusions derived form the flap mode apply for the edge-wise and torsion modes. The edge-wise shows a strong dependence with the Mach number while for the torsion mode the contribution of more ICs is needed to reconstruct the work-per-cycle.

6. Unsteady pressure of airfoils located far away from the central vibrating airfoil is controlled by acoustic perturbations. 


\section{ACKNOWLEDGMENTS}

The authors wish to thank Industria de Turbopropulsores S.A. for allowing the publication of this paper and for its support during the project. This work has been partially funded under grant DPI2009-14216 by the Spanish Minister of Education and Science and by the FUTURE project of the European Commission under Contract ACP7-GA-2008-213414.

\section{References}

[1] Corral, R., Gallardo, J., and Vasco, C., "Aeroelastic Stability of Welded-in-Pair Low Pressure Turbine Rotor Blades: A Comparative Study Using Linear Methods," Journal of Turbomachinery, Vol. 129, January 2007, pp. 72-83.

[2] Corral, R., Gallardo, J. M., and Martel, C., "A Conceptual Flutter Analysis of a Packet f Vanes Using a Mass-Spring Model," Journal of Turbomachinery, Vol. 131, April 2009, pp. 021016-1-7.

[3] Buffum, D. and Fleeter, S., "Aerodynamics of a Linear Oscillating Cascade,” TM 103250, NASA, 1990.

[4] He, L., "Unsteady flow in oscillating turbine cascades : Part 2-Computational study," Journal of Turbomachinery, Vol. 120, No. 2, 1998, pp. 269-275.

[5] Nowinski, M. and Panovsky, J., "Flutter Mechanisms in Low Pressure Turbine Blades," Journal of Engineering for Gas Turbines and Power, Vol. 122, 2000, pp. 89-98.

[6] Bolcs, A. and Fransson, T., "Aeroelasticity in Turbomachines: Comparision of Theoretical and Experimental Cascade Results," Tech. rep., Laboratoire de Thermique Appliquee et de Turbomachines, EPFL, 1986.

[7] Corral, R., Escribano, A., Gisbert, F., Serrano, A., and Vasco, C., "Validation of a Linear Multigrid Accelerated Unstructured Navier-Stokes Solver for the Computation of Turbine Blades on Hybrid Grids," AIAA Paper 2003-3326, 9th AIAA/CEAS Aeroacoustics Conference, Hilton Mead, South Carolina, May 2003.

[8] Corral, R. and Gallardo, J., "Verification of the Vibration Amplitude Prediction of Self-Excited LPT Rotor Blades Using a Fully Coupled Time-Domain Non-Linear Method and Experimental Data," ASME Paper 2008-GT-51416, Proceedings of the 51st ASME Gas Turbine and Aero engine Congress, Exposition and Users Symposium, Berlin, Germany, June 2008.

[9] Corral, R. and Gallardo, J., "Non-Linear Dynamics of Multi-Mode Unstable Bladed-Disks: Part I - Description of a Canonical Model and Phenomenological Results," ASME Paper 2011-GT-22943, 2011.

[10] Burgos, M., Corral, R., and Contreras, J., "Efficient Edge Based Rotor/Stator Interaction Method," AIAA Journal, Vol. 41, No. 1, January 2011, pp. 19-31.
[11] Corral, R. and Gisbert, F., "A Numerical Investigation on the Influence of Lateral Boundaries in Linear Vibrating Cascades," Journal of Turbomachinery, Vol. 125, July 2003, pp. 433-441.

[12] Panovsky, J. and Kielb, R., "A Design Method to Prevent Low Pressure Turbine Blade Flutter," Journal of Engineering for Gas Turbines and Power, Vol. 122, 2000, pp. 89-98. 